\title{
ERRATA, VOLUME 61
}

S. C. Chang, Contributions to projective theory of singular points of space curves.

p. 369, line after display (2). Read " $\alpha_{10}$ " for " $\alpha_{01 .}$ "

Samuel Eilenberg, Homology of spaces with operators. I.

p. 382, line 15. After " $(a, U)$ " insert "and their intersections."

p. 382, Lemma 3.1. The lemma should read "The homomorphism $\phi^{*}$ is continuous."

The proof of the strong continuity as given in the text contains an error. As a result Corollary 3.2 should be suppressed. All subsequent references to strong continuity should be replaced by statements concerning continuity only. Thus isomorphisms (12.4), (16.4), (16.5), (16.6), (17.9) are algebraic isomorphisms continuous in one direction. In order to be able to assert that the isomorphisms are topological an additional argument concerning the continuity of the inverse map is needed. In the case of (16.4) and (16.5) a continuous inverse is actually constructed. In the other cases, the construction of a continuous inverse can readily be supplied. If the coefficient group is compact, the construction of a continuous inverse is superfluous.

p. 390, line 12. Read " $\sum_{i=0}^{\mathfrak{a}}$ " for " $\sum_{i=0}^{b}$ "

G. K. Kalisch. On special Jordan algebras.

p. 490 , line 7. Read " $h_{k i}$ " for " $h_{k i t}^{T}$."

p. 490 , line 8 . Read " $h_{k 1}^{T}$ " for " $h_{k 1 .}$."

p. 490, line 8. Read "with similar results for $h_{k 1}^{T}$ " for "with similar results for $h_{k 1 .}$ " 